\title{
Electron microscopy characterization of W-O multilayers
}

\author{
N.M.G. Parreira* T. Polcar*** Y.T. Pei*** D. Galvan*** J.Th.M. De Hosson*** and A. \\ Cavaleiro* \\ * SEG-CEMUC, Mechanical Eng Department, University of Coimbra, Portugal \\ ** Faculty of Electrical Eng., Czech Technical University Prague, Czech Republic \\ *** Department of Applied Physics, Materials Innovation Institute (M2i), University of Groningen, \\ The Netherlands \\ albano.cavaleiro@dem.uc.pt
}

In the decorative field, besides the optical characteristics needed for achieving desired colours, the material should have suitable properties able to assure the daily use of the component without significant degradation [1]. Oxides are well known materials with a high wear and corrosion resistance which make them a preferential choice of coatings for environmental protection. However, the intrinsic colours possible to be achieved in a single oxide system are very limited. For example in W-O system, besides the different tonalities of grey, only the dark blue can be obtained for slightly substochiometric $\mathrm{WO}_{3}$ compound [2]. The pallet of colours exhibited by $\mathrm{WO}_{3}$ coatings is due to interferometric effects. Recently, it was shown that playing with the gradient of the chemical composition of W-O layers it was possible to tune the global colour of the coatings, which made this system an attractive candidate for decorative applications [3].

In this study the microstructural characterization of $\mathrm{W}-\mathrm{O}$ coatings by transmission and scanning electron microsocopies (TEM and SEM, respectively) are presented. The coatings were deposited by reactive magnetron sputtering in RGPP mode (reactive gas pulsing process) [4]. SEM micrographs show the multilayer character of the deposited coatings whatever the pulsing parameters used in the deposition were $\left(\mathrm{T}\right.$ - period, and $\mathrm{t}_{\mathrm{ON}}$ - injection time) [5].

X-ray diffraction revealed that the structure of the coatings deposited by RGPP was in agreement to the multilayer effect, i.e. the detected phases were related to the $\mathrm{W}$-rich and the O-rich layers forming the stacking [5]. All the coatings exhibited a mixture of $\alpha-\mathrm{W}, \beta-\mathrm{W}_{3} \mathrm{O}$ and quasi-crystalline $\mathrm{W}-\mathrm{O}$ (a broad peak centred at $2 \theta \approx 25^{\circ}$, corresponding to the more intense peaks of $\mathrm{WO}_{3}$ ) phases attributed to the $\mathrm{W}$ layer, a transition layer and the compound layer, respectively [5]. Using TEM, even in high-resolution mode, only 2 layers are possible to be distinguished (Fig. 1a), and the $\beta-\mathrm{W}_{3} \mathrm{O}$ phase could hardly be observed (see Fig. 1b), although electron diffraction (insert of Fig. 1a) confirmed its existence. The W-rich layer showed a fairly crystalline structure in comparison with the featureless aspect of the nanocrystalline O-rich layer.

In order to distinguish and calculate the extension of each of the layers that form the stacking, the cross section of the coatings was analysed by energy filtering images (GIF) which allows evaluating the $\mathrm{O}$ concentration across the film thickness. The GIF images were obtained by subtraction of two images taken before and after the O-K edge defined at $530.6 \mathrm{eV}$, i.e. at $-15 \mathrm{eV}$ and $+15 \mathrm{eV}$, respectively. Thus, the new image was generated by the electrons with energy between 515.6 and $545.6 \mathrm{eV}$ as shown in Fig. 2a. By integration of the intensities in a selected area of Fig. 2a, the oxygen distribution across the coating thickness can be accessed (Fig. 2b). In this particular case, the definition of the stacking layers, including the transition layer, can be achieved, allowing to calculate their thickness, even if they are not perfectly distinguished by HRTEM observation. For example, in this case the transition layer is about $45 \mathrm{~nm}$ thickness. This information is revealed to be 
fundamental for modelling the optical behaviour of the multilayer, since other characterization techniques (e.g. Auger spectroscopy analysis after progressive in-depth etching of the sample) do not allow to establish directly the correspondence between the chemical composition and the depth at which the analysis is being performed [3].

\section{References}

[1] U. Beck, G. Reiners, U. Kopacz, H.A. Jehn, Surface and Coatings Technology 60 (1993) 389.

[2] A. Yamamoto, Y. Abe, M. Kawamura, K. Sasaki, Vacuum 66 (2002) 269.

[3] T. Polcar, T. Kubart, E. Malainho, M.I. Vasilevskiy, N.M.G. Parreira, A. Cavaleiro, Nanotechnology 19 (2008) 395202.

[4] N.M.G. Parreira, T. Polcar, A. Cavaleiro, Plasma Processes and Polymers 4 (2007) 62.

[5] N.M.G. Parreira, T. Polcar, A. Cavaleiro, Surface and Coating Technology 201 (2006) 5481.
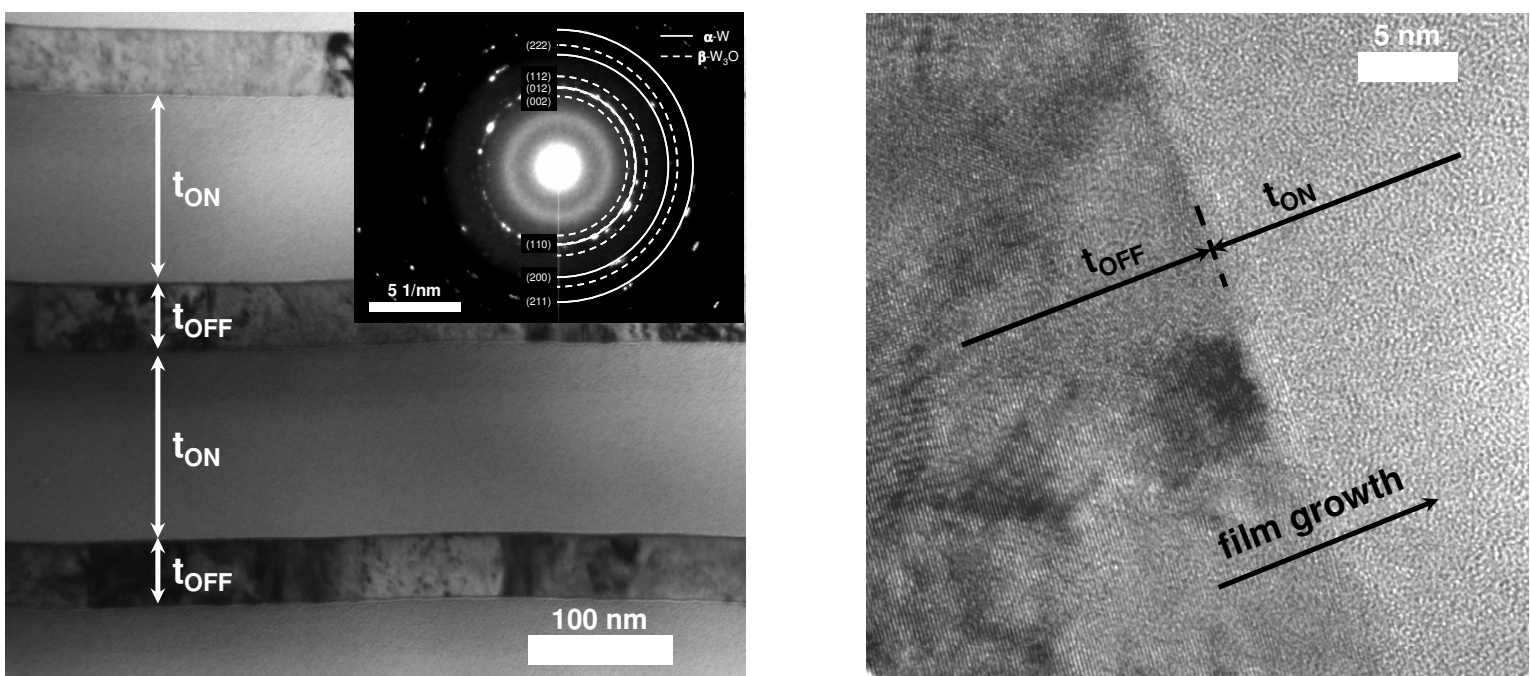

Fig. 1. (a) Cross-section bright field TEM image with selected area electron diffraction pattern in the insert and (b) HR-TEM image of a W-O coating deposited by RGPP ( $\mathrm{T}=50 \mathrm{~s}$ and $\left.\mathrm{t}_{\mathrm{ON}}=25 \mathrm{~s}\right)$.
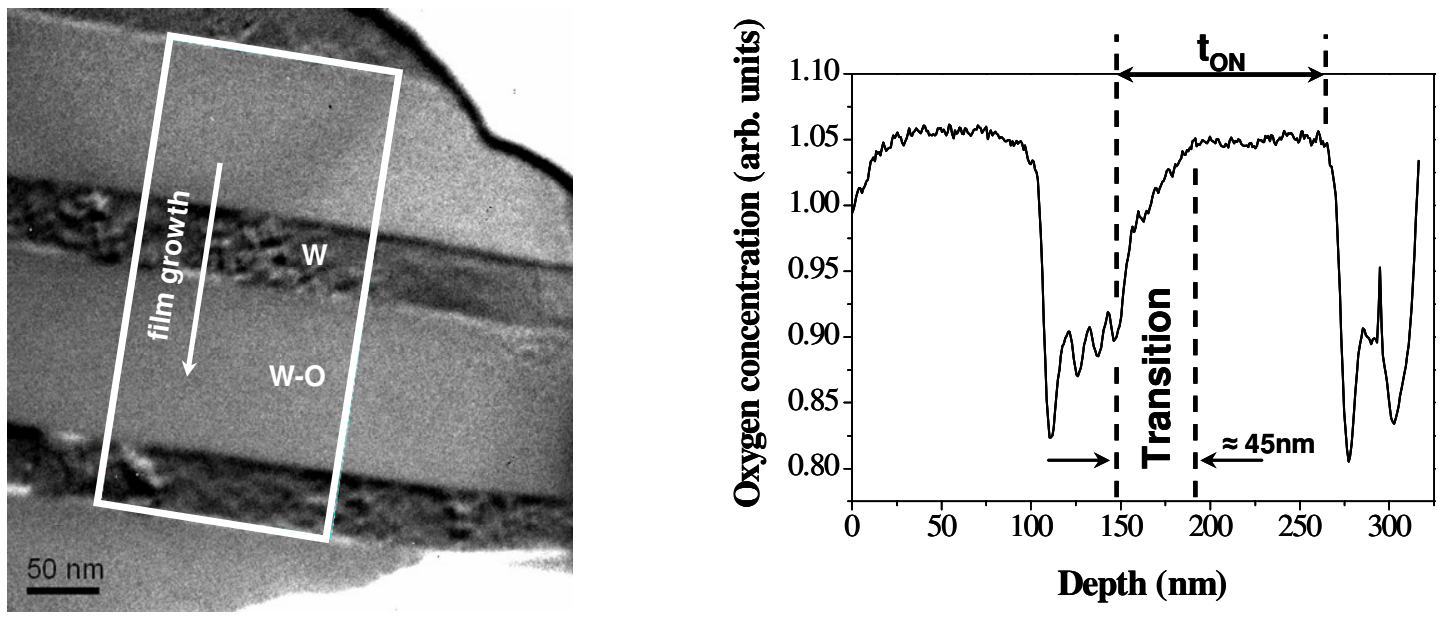

Fig. 2. (a) Oxygen map image obtained from energy filtered TEM of the O-K energy of the coating $\mathrm{WO}_{50-25}$ and (b) integration of the intensities from the selected area in (a). 